\title{
Cell Segmentation of 2D Phase-Contrast Microscopy Images with Deep Learning Method
}

\author{
Aydin Ayanzadeh, Hüseyin Onur Yağar \\ Informatics Institute, Ayazaga Campus, \\ Istanbul Technical University, \\ Istanbul, Turkey \\ \{ayanzadeh17, yagar19\}@itu.edu.tr
}

\author{
Özden Yalçın Özuysal, Devrim Pesen Okvur \\ Department of Molecular Biology and \\ Genetics, Izmir Institute of Technology, \\ Izmir, Turkey \\ \{ozdenyalcin, devrimpesen\}@iyte.edu.tr
}

\author{
Behçet Uğur Töreyin \\ Informatics Institute, Ayazaga Campus, \\ Istanbul Technical University, \\ Istanbul, Turkey \\ toreyin@itu.edu.tr
}

\author{
Devrim Ünay \\ Biomedical Engineering, Faculty of \\ Engineering, Izmir University of Economics, \\ Izmir, Turkey \\ unaydevrim@gmail.com
}

\author{
Sevgi Önal \\ Biotechnology, Izmir \\ Institute of Technology, \\ Izmir, Turkey \\ sevgional1@gmail.com
}

\begin{abstract}
The quantitative and qualitative ascertainment of cell culture is integral to the robust determination of the cell structure analysis. Microscopy cell analysis and the epithet structures of cells in cell cultures are momentous in the fields of the biological research process. In this paper, we addressed the problem of phase-contrast microscopy under cell segmentation application. In our proposed method, we utilized the state-of-theart deep learning models trained on our proposed dataset. Due to the low number of annotated images, we propose a multiresolution network which is based on the U-Net architecture. Moreover, we applied multi-combination augmentation to our dataset which has increased the performance of segmentation accuracy significantly. Experimental results suggest that the proposed model provides superior performance in comparison to traditional state-of-the-art segmentation algorithms.
\end{abstract}

Keywords-Deep learning, phase-contrast microscopy, cell segmentation.

\section{INTRODUCTION}

Phase-contrast microscopy is particularly important in biology. It reveals many of cells structure that are not visible with a simpler microscope. Hence, this microscopy structure is one of the challenging microscopy imaging which has been encountered scientists in segmentation and tracking tasks. The main reason for this is the region of interests(ROIs) color can not be realized and extracted from background efficiently. Therefore, most of the segmentation and tracking tasks does not have enough qualification for satisfying the robustness, completeness and accuracy factors. Therefore, we need to apply different technical pre-processing for increasing the visibility of the ROI. One of this method which can increase the brightness of ROI is histogram quantization which can help in the motility of the cells besides of applied methods.

Live-cell microscopy imaging together with appropriate image analysis tools have the potential to aid biologists in quantifying biological phenomena. For instance, without proper tools

978-1-7281-2420-9/19/\$31.00 @2019 IEEE for segmentation tasks of cells, scrutinizing the properties of the cells in time-lapse microscopy could be a time consuming and tedious task for biologists. Automatic segmentation tools vary and include: automatic gray-level thresholding[1], the watershed algorithm[2] and Active Contours[3].

Jaccard, Nicolas, et al.[4] presented a method based on image-processing approach by local contrast thresholding, which has robust results on their presented PCM dataset but it can not satisfy the completeness and robustness factors on the adjacent or touching cells. In contrast to other convenient methods such as Convolutional Neural Networks which outperform the traditional cell segmentation solutions[5], Arbelle, et al.[6] proposed the concept of Generative Adversarial Networks for microscopy cell segmentation. Moreover, in[8], an integration of Convolutional Long Short Term Memory (C-LSTM) into a U-Net[7] is presented and they evaluate the combination of the presented model. HF. Tsai, et al.[9] proposed the architecture based on a Mask R-CNN model on phase-contrast microscopy images for cell segmentation application, but the trained model is not transferable to domain of dataset which is different from that in the train set and it needs a very high computation to reach acceptable results.

Our contribution has three folds: 1) We extended the concept of multi-resolution network which is based on U-Net for segmentation of cells in two-photon microscopy images. 2) We proposed a novel data augmentation which utilizing the sequential transformation for our input dataset which increases the accuracy of segmentation in comparison to the conventional U-Net[7] and Empirical Gradient Threshold (EGT)[10] which we represent them in evaluation section. 3) We achieve the high accuracy of segmentation with a low number of training examples, Therefore, our propose approach dramatically reduce the amount of manual workload.

The rest of the paper is organized as follows. In SectionII, we present the details of sample preparation and imaging. In sectionIII, we define the problem and elaborates on the 
proposed solution. SectionIV presents the results for both common and our proposed approach. SectionV, concludes the paper and suggests possible extensions for future work.

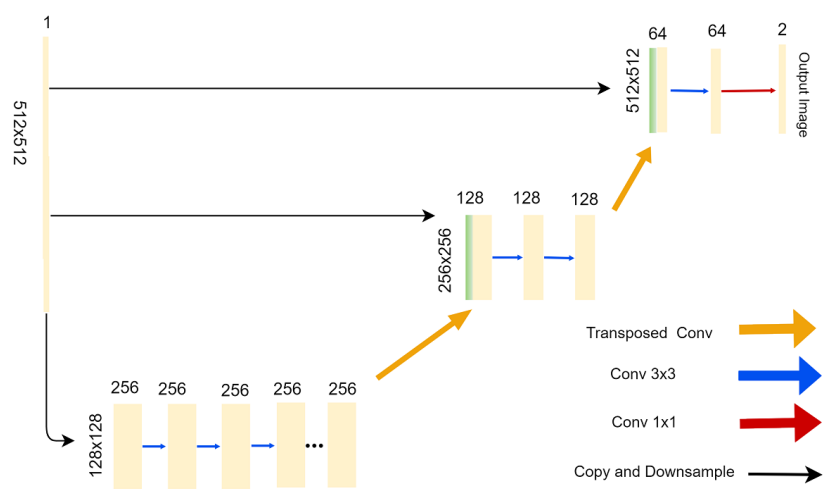

Fig. 1: Schematic structure of our proposed architecture.

\section{DATASET PREPARATION}

Three breast cell lines with different morphology and mobility were used in this workspace, invasive breast cancer cell line MDA-MB-231 in mesenchymal morphology; non-invasive breast cancer cell line MCF7 in epithelial morphology and normal breast cell line MCF10A in epithelial morphology. MDA MB 231 and MCF7 cells were grown in DMEM medium containing $10 \%$ fetal bovine serum while MCF10A cells were in DMEM:F12 (1:1) containing 5\% horse serum, $20 \mathrm{ng} / \mathrm{ml}$ epidermal growth factor, $500 \mathrm{ng} / \mathrm{ml}$ hydrocortisone, $100 \mathrm{ng} / \mathrm{ml}$ cholera toxin, and $10 \mu \mathrm{g} / \mathrm{ml}$ insulin. The cells were grown in incubators containing $5 \% \mathrm{CO} 2$ and humidity at $37^{\circ} \mathrm{C}$. In terms of viability, proliferation, and infection, they were monitored daily by inverted light microscopy and multiplied with trypsin, paying attention to the doubling times of each cell culture. Periodic stocking was applied to ensure the continuity of the cells. Cells were frozen in a solution containing FBS and DMSO. Storage was carried out at $-80^{\circ} \mathrm{C}$ and then $-196^{\circ} \mathrm{C}$ in a liquid nitrogen tank. Then, by utilizing the Fiji distribution of ImageJ[12,13] individual cell boundaries are marked for images taken for single-cell analysis and cell layer boundaries are be manually marked for images analysis.

About the data records, our presented dataset consists of the following: 600 phase-contrast microscope images (TIFF format, $2568 \times 1912$ pixels, 16 Bit depth) where 25 of them are annotated by an expert, 18 of annotated images are utilized in training set and rest of them are used in test at evaluation process. Due to the low-quality conditions of the cell images in the level of contrast, we increase the signal-tonoise ratio(SNR) by adjusting the contrast of the image and normalize the images that changes the range of pixel intensity values.

\section{METHOD}

\section{A. Multi-Resolution Network}

U-Net is an encoder-decoder architecture which is very efficient in training and generalizing with small types of

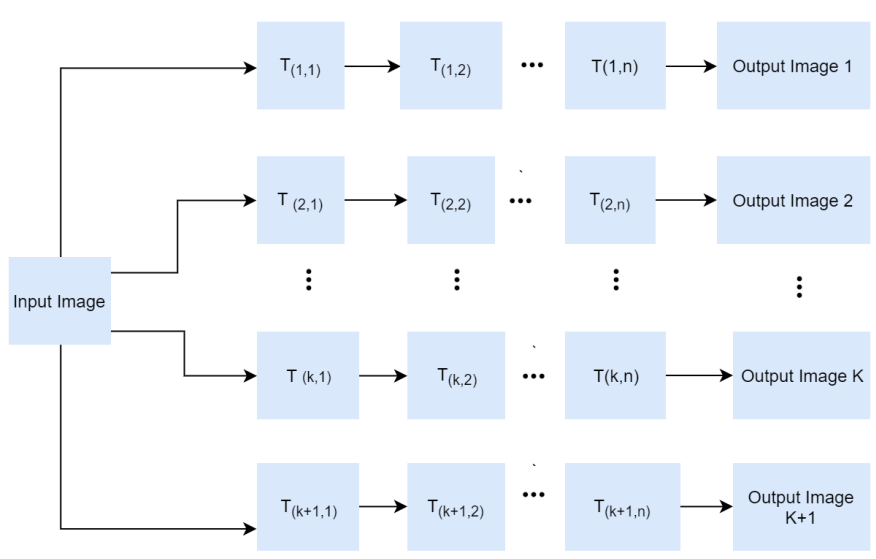

Fig. 2: The Schematic architecture of proposed augmentation method on presented deep learning network.

dataset. However, modifying the U-Net according to the type of dataset is an interesting field of study on phase-contrast microscopy images. This architecture has high capacity of extension in different aspects. In Fig1, the schematic of the proposed architecture is presented.

The proposed network was trained with 18 annotated images for training sets. This network is composed of a CNN with $3 \times 3$ convolution filters and applied leaky ReLU as an activation function in our application. To keep the spatial size of the input image unchanged, we utilized padding layer in this architecture and each layer of this are proceeded by batch normalization. Due to the structure of our presented dataset, the lowest resolution segment of the network size is $128 \times 128$ pixels which is downscaled from original size. This fold consists 15 convolution layers with 256 channels where applying consecutive convolution layer makes the model effective in congregating of outputs based on the extracted features[11]. To reach the resolution of $256 \times 256$ pixels from the lowest resolution, a transposed convolution with stride of 2 is applied to increase the resolution of the input. The last section contains full resolution fold with $512 \times 512$ pixels which have 2 layers with 64 channels and the final layer for output image.

\section{B. Data Augmentation}

Data Augmentation is very common in classification application and it can increase the accuracy of classification. Most of Deep Neural Networks(DNN) are structured to be invariant on specific geometrical transformation, which can not extract new information from generated images. We propose a novel type of augmentation which is based on combining the sequential augmentation in the dataset during the training process. Schematic structure of our presented workflow is shown in Fig2. As an input image $\mathrm{T}_{(\mathrm{K}, \mathrm{n})}$ which $\mathrm{K}$ represents the number of augmentation and $\mathrm{n}$ indicates the number of transformation in augmentation process. In augmentation procedure, input image receives the specific transformation and after applying the sequence of transformations, the output images are merged to create new output from the previous transformed images. In this paper, we utilized $n=3$ for the 


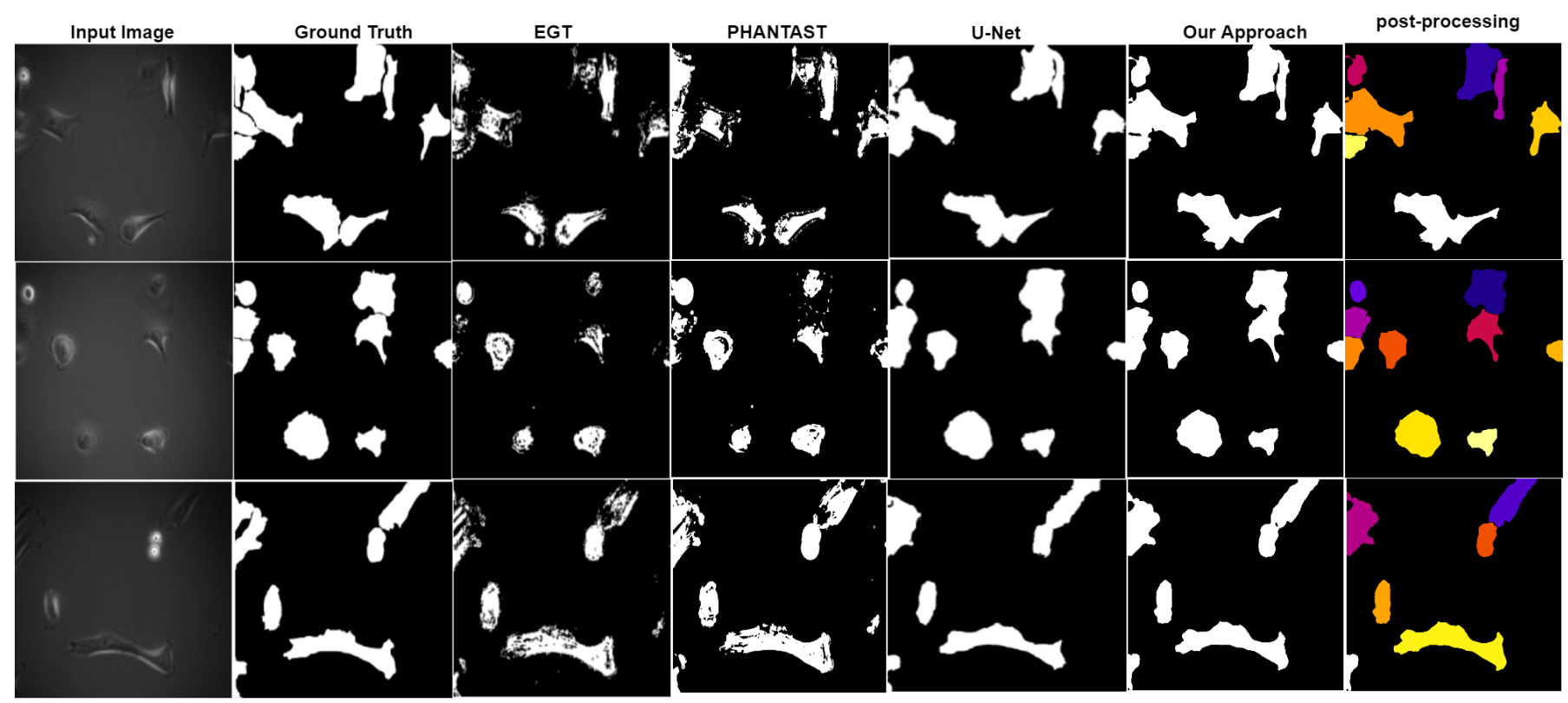

Fig. 3: Results of the segmentation for three frames of breast cancer MDA-MB-231 dataset. The first and second columns are the input images and ground truth in determined frames, the third and fourth column is the results of the EGT and PHANTAST respectively. Fifth column represents the results of U-Net and last two columns depict our proposed approach without post-processing and after applying the Distance Transform Watershed; moreover, Connected Component Labeling on the last column shows the level of separation on adjacent or touching cells.

number of transformation in each augmentation and $\mathrm{k}=4$ for the number of augmentation with different configuration for each input image.

The main utilized Transformations contain elastic transform, rotation and translation with different configuration. Due to the low number of annotated images for segmentation task, we used both of the combined and single augmentation in our work to enlarging the number of the training set. The proposed augmentation can efficiently simulate the shape of the cells on unseen images and it increase the accuracy of segmentation significantly.

\section{RESUlts}

To scrutinize the accuracy of the proposed method, it should be analyzed with quantitative and qualitative perspectives. The method was evaluated using the Dice Coefficient, F-score and Intersection over Union (IoU) scheme which quantitative results of the cell segmentation are shown in Table I. The qualitative results of segmentation is also shown in Fig3.

\section{A. Implementation Details}

We utilize PyTorch framework[14] in the implementations and all of the experiments were done on a single NVIDIA TitanX with 12 GB memory. As a training regime, our model was trained with Adam optimizer with learning rate of $2 \times 10^{-4}$. To schedule the learning rate, we followed the stepwise decay for which the amount of learning rate is reduced with the multiplicative factor of 0.8 when our training loss does not decrease in every 20 epochs; The batch size was applied 8 in our training regime. The loss is defined using the distance weighted cross-entropy loss which was proposed in the U-Net article[7]. The presented loss function is the thrust cell separation with a specific type of penalty factor which has exponential behavior. Additionally, the pixels which are located between the touching and adjacent have a higher impact on loss rather than the far pixels which is not located between the boundary of the cells.

\section{B. Evaluation Metrics}

Comparison between the algorithm output and the ground truth was realized using the following well-known evaluation metrics. IoU is indicated in (1) and F-score is the harmonic mean of precision and recall which is shown in (2) as follows:

$$
I o U(X, Y)=\frac{|X \bigcap Y|}{|X \bigcup Y|}
$$

$$
F-\text { Score }=\frac{2 \cdot \text { precision } \cdot \text { recall }}{\text { precision }+ \text { recall }}
$$

The third evaluating metric is Dice Coefficient which measures the accuracy of spatial overlap between the ground truth and predicted image which is indicated in (3) as follows:

$$
\operatorname{Dice}(X, Y)=\frac{2|X \cdot Y|}{|X|+|Y|}
$$


TABLE I: Quantitative results of cell segmentation

\begin{tabular}{|c|c|c|c|}
\hline Methods & IoU & Dice Coefficient & F-Score \\
\hline Emperical Gradient Threshold & 0.381 & 0.578 & 0.547 \\
\hline PHANTAST & 0.597 & 0.651 & 0.673 \\
\hline U-Net & 0.825 & 0.854 & 0.837 \\
\hline Proposed Method & $\mathbf{0 . 8 7 1}$ & $\mathbf{0 . 8 9 9}$ & $\mathbf{0 . 8 8 1}$ \\
\hline
\end{tabular}

\section{Experimental Results}

We present experimental results of our approach compared to different baselines. Our proposed model achieves state-ofthe-art performance in our presented dataset. In what follows, we compare the results of our dataset with different baseline. The results of the utilized methods are shown in Fig3. We achieve a mean Intersection over Union (mIoU) of $\mathbf{0 . 8 7}$, which is noticeably better in compare of baseline. Additionally, our proposed approach is successful in predicting of the shapes of the cells in comparison to the U-Net and other baselines. In the case of baseline, often fail to be segmented and contribute to higher mIoU and the level of segmentation in EGT and PHANTAST is mediocre(mIoU 0.38 and 0.59). Moreover, EGT and PHANTAST are not precise in the estimation of the cell boundaries. Obtained results in Table I are applied on the test-set using the F-score, IoU and Dice Coefficient for comparison purposes. The same approach was applied to a previously described PCM image segmentation algorithms such as PHANTAST, EGT, and U-Net in comparison of our proposed approach. The results are shown as the average scores over the test set. Additionally, we applied the Distance Transform Watershed[15] as a post-processing method to separate the touching cells. As method configuration, after normalizing the weight, for diagonal and orthogonal neighbors, we set 2 and 1 consecutively. To have better visualization, Connected Component Labeling is applied after utilizing the determined post-processing to show the separation of the cells in the output image. Applying determined approach achieves IoU to 0.891 in accuracy. The last column of the Fig3 represents the visualization of Distance Transform Watershed as postprocessing on our method.

\section{CONCLUSiON AND FUture WORK}

We presented a deep learning approach for cell segmentation in 2D phase contrast microscopy images with specific type of data augmentation. Given the significant variability in cell appearance that resulted from using different stains and different cell types, achieving robust cell segmentation with the simple Empirical Gradient Threshold is difficult. The results show that our proposed deep learning based approach outperforms the state-of-the-art algorithms (EGT, PHANTAST, and U-Net) in robustness, completeness and accuracy. As future work, we plan to extend the dataset by increasing manual annotations for not only segmentation but also tracking of cells. Then onwards we will fortify our analysis by constructing lineage relationships to provide information about cell behavior.

\section{SUPPLEMENTARY INFORMATION}

Datasets generated and analyzed in this paper are provided as an additional file in https://doi.org/10.6084/m9.figshare.8965820, You can cite our dataset by writting below content in your work.

Ayanzadeh, Aydin; Yă̆ar, Hüseyin Onur; Özuysal, Özden Yalçın; Okvur, Devrim Pesen; Töreyin, Behçet Ŭ̆ur; Ünay, Devrim; et al. (2019): Phase Contrast Microscopy of cells with annotation. figshare. Dataset.

\section{ACKNOWLEDGMENT}

The data used in this study is collected under the Marie Curie IRG grant (no: FP7 PIRG08-GA-2010-27697).

Aydin Ayanzadeh's work is supported, in part, by Vodafone Turkey, under project no. ITUVF20180901P04 within the context of ITU Vodafone Future Lab R\&D program.

This work is in part funded by İTÜ BAP MGA-2017-40964

\section{REFERENCES}

[1] T. Kanade, et al., "Cell image analysis: Algorithms, system and applications," in WACV. IEEE, 2011, pp. 374-381.

[2] L. Vincent and P. Soille, "Watersheds in digital spaces: an efficient algorithm based on immersion simulations," IEEE PAMI, vol. 13, no. 6, pp. 583-598, 1991.

[3] P. Bamford and B. Lovell, "Unsupervised cell nucleus segmentation with active contours," Signal Processing, vol. 71, no. 2, pp. 203-213, 1998.

[4] Jaccard, Nicolas, et al. "Automated method for the rapid and precise estimation of adherent cell culture characteristics from phase contrast microscopy images." Biotechnology and bioengineering 111.3 (2014): 504-517.

[5] O. Z. Kraus, J. L. Ba, and B. J. Frey, "Classifying and segmenting microscopy images with deep multiple instance learning," Bioinformatics, vol. 32 , no. 12 , pp. i52-i59, 2016

[6] A. Arbelle and T. Riklin Raviv, "Microscopy cell segmentation via adversarial neural networks," arXiv preprint arXiv:1709.05860, 2017.

[7] Ronneberger, Olaf, Philipp Fischer, and Thomas Brox. "U-net: Convolutional networks for biomedical image segmentation." International Conference on Medical image computing and computer-assisted intervention. Springer, Cham, 2015.

[8] Arbelle, Assaf, and Tammy Riklin Raviv."Microscopy cell segmentation via convolutional LSTM networks." 2019 IEEE 16th International Symposium on Biomedical Imaging(ISBI 2019).IEEE, 2019.

[9] Tsai, Hsieh-Fu, et al. "Usiigaci: Instance-aware cell tracking in stain-free phase contrast microscopy enabled by machine learning." SoftwareX 9 (2019): 230-237.

[10] Chalfoun, Joe, et al. "Empirical gradient threshold technique for automated segmentation across image modalities and cell lines." Journal of microscopy 260.1 (2015): 86-99.

[11] Long, J., Shelhamer, E., Darrell, T.: Fully convolutional networks for semantic segmentation (2014), arXiv:1411.4038 [cs.CV]

[12] Schindelin, J. et al. Fiji: An open-source platform for biological-image analysis. Nature Methods 9, 676-682 (2012)

[13] Schneider, C. A., Rasband, W.S.Eliceiri, K. W. NIH image to ImageJ: 25 years of image analysis. Nature Methods 9 671-675 (2012).

[14] A.Paszke, S.Gross, S.Chintala, G.Chanan, E.Yang, Z.DeVito, Z. Lin, A.Desmaison, L.Antiga, and A.Lerer.Automatic differentiation in pytorch. In NIPS Workshop, 2017.

[15] Acharjya, P. P., et al. "A new approach of watershed algorithm using distance transform applied to image segmentation.” International Journal of Innovative Research in Computer and Communication Engineering 1.2 (2013): 185-189. 